\title{
BMJ Open Addressing the psychological impact of COVID-19 on healthcare workers: learning from a systematic review of early interventions for frontline responders
}

To cite: Hooper JJ, Saulsman L, Hall T, et al. Addressing the psychological impact of COVID-19 on healthcare workers: learning from a systematic review of early interventions for frontline responders. BMJ Open 2021;11:e044134. doi:10.1136/ bmjopen-2020-044134

- Prepublication history for this paper is available online. To view these files, please visit the journal online (http://dx.doi. org/10.1136/bmjopen-2020044134).

Received 24 August 2020 Revised 27 February 2021 Accepted 22 April 2021

Check for updates

(c) Author(s) (or their employer(s)) 2021. Re-use permitted under CC BY-NC. No commercial re-use. See rights and permissions. Published by BMJ.

${ }^{1}$ School of Psychological Science, The University of Western Australia, Crawley, Western Australia, Australia ${ }^{2}$ Clinical Research Centre Gascoyne House, West Wing, Graylands Hospital, Mount Claremont, Western Australia, Australia

Correspondence to Ms Jasmine Jean Hooper; jasminejhooper@gmail.com

\section{ABSTRACT}

Objectives Protecting healthcare workers from psychological harm is an urgent clinical issue within the current COVID-19 pandemic. Research on early psychological programmes that aim to prevent or reduce mental health symptoms and that have been tested in frontline responders may assist service providers with choosing a suitable intervention for rapid dissemination in healthcare settings.

Design and outcome measures First, Embase, Web of Science, PsycINF0 and Google Scholar were searched through a systematic literature review of early psychological interventions administered to frontline responders in the last 15 years. Interventions were included if they were designed to prevent or reduce psychological impact and had outcome measures of psychological distress (eg, general psychopathology, post-traumatic stress disorder and stress) and/or positive mental health domains (eg, resilience, self-efficacy and life satisfaction). Second, the suitability of these programmes for the healthcare workforce was evaluated according to the criteria of effectiveness, content applicability and feasibility.

Results 0 f 320 articles retrieved, 12 relevant studies were included that described six early psychological interventions. Although the evidence base is limited, psychological first aid, eye movement desensitisation and reprocessing, and trauma risk management showed effectiveness across at least two studies each with frontline workers. Resilience and coping for the healthcare community; anticipate, plan, and deter; and resilience at work programmes found promising results in single studies. Concerning other suitability criteria, all programmes appear applicable to healthcare settings and have acceptable feasibility for rapid implementation. Conclusions Despite the limited evidence, several interventions were identified as potentially suitable and useful for improving psychological functioning of healthcare workers across a variety of disaster situations. Service providers should continue to implement and evaluate early psychological interventions in frontline workers in order to refine best practices for managing the psychological impact of future disasters.
Strengths and limitations of this study

- This is a timely review given the current COVID-19 crisis and the limited availability of evidence-based information on early psychological interventions for healthcare workers and other frontline responders.

- Practical suitability of each programme was carefully considered, to address the need for rapid and widespread implementation of psychological support in the healthcare workforce.

- Despite multiple databases searched and a rigorous review process, it is possible that there are other suitable programmes not identified by this review paper.

- Given the chaotic nature of healthcare services when dealing with mass trauma or disasters, there may also be existing programmes that have not yet received formal evaluation.

\section{INTRODUCTION}

Healthcare decision-makers are continually seeking information on how to provide the best psychological assistance to workers. In particular, working in the frontline during local and global disasters involves repeated exposure to traumatic events, which can have a major impact on mental health such as increased rates of acute stress disorder, posttraumatic stress disorder (PTSD), anxiety and depression. ${ }^{12}$ Continued psychological distress may lead to further adverse outcomes of substance abuse and suicide risk ${ }^{3}$ as well as burnout, compassion fatigue and secondary traumatisation. ${ }^{45}$ In contrast, positive mental health domains such as resilience may serve to protect the mental health of frontline responders. ${ }^{6}$ In many cases, psychological harm may have a delayed onset, with symptoms only developing several months or years after the traumatic event. ${ }^{7}$ It is therefore important for healthcare services to 
implement effective early intervention measures that seek to mitigate ongoing psychological distress and minimise the development of post-traumatic symptoms in the workforce. $^{8}$ However, organisations require evidence-based information about available psychological programmes before they can make well-informed decisions on how to assist the mental health needs of their staff. By reviewing this information, the current study seeks to guide the response of healthcare service providers during current and future disasters.

The novel SARS-CoV-2 or COVID-19 pandemic ${ }^{9}$ has led to a sharp increase in demand for frontline health and social care workers such as nurses, doctors, paramedics, and forensic workers as well as other security personnel including police officers and the military. ${ }^{10}$ This increased demand occurred in the context of chronic shortages in frontline staff due to quarantine restrictions, sick leave, and the need for staff to continue caring for dependents and attend to other family responsibilities. ${ }^{11}$ As a result, these frontline responders have elevated workloads and are expected to work long hours under highly stressful conditions. COVID-19 has brought further workplace stressors to healthcare workers due to fears of contracting the virus and infecting others, difficulties accessing personal protective equipment, stigma, discrimination from the public and heightened emotional burden. ${ }^{12}$ These stressors have triggered elevated rates of psychological distress in healthcare workers such as depression, anxiety, and PTSD ${ }^{1314}$ and may indeed have secondary effects such as burnout and compassion fatigue, creating concern over a subsequent mental health crisis. ${ }^{15}$

Healthcare services have responded swiftly to mental health concerns in the workforce. Initial assistance has included organisation-wide provision of nonpsychological practical help through infection control procedures, access to protection equipment and response to other basic physical needs. ${ }^{16}$ There is also a wealth of ad-hoc stress management instructions available online to healthcare workers during COVID-19, which provide lists of basic educational information about psychological self-care and help-seeking. Staff are encouraged to contact personal crisis helplines and professional support services if mental health symptoms persist, with cognitive-behavioural therapy (CBT) interventions (eg, trauma-focused CBT) endorsed as an effective long-term treatment option for the reduction of ongoing psychological symptoms. ${ }^{11} 1718$ However, such individualised interventions carry inherent limitations, as they require active help-seeking behaviour and stigma regarding mental health has been identified as a substantial barrier to seeking psychological support among healthcare workers. ${ }^{19}$

The provision of early psychological interventions to all trauma-exposed individuals may be equally as important as delayed intervention after symptom onset, in the context of preventing and minimising the shortterm and long-term negative impacts of traumatic events on mental health. ${ }^{20}$ Recent research has recommended that frontline responders should receive early psychological intervention within the first few months of the traumatic event. ${ }^{21}{ }^{22}$ However, there is currently no consistent implementation of early psychological interventions among trauma-exposed workforces. One major barrier to implementation is the lack of accessible information regarding early psychological interventions suitable for frontline workers. Furthermore, there is a paucity of research that has tested the effectiveness of these early interventions specifically within healthcare providers. Despite obvious differences in job demands across various frontline services and disaster situations, these workers all face frequent trauma exposure within the workplace. ${ }^{45}$ A potential solution to this issue is therefore to examine psychological programmes that have been administered and tested in all frontline responders during previous disasters and pandemics and review whether these programmes are relevant and practical for rapid implementation within healthcare services. The key objective of this paper was therefore to provide evidence-based and practical information to assist healthcare service providers in deciding how best to protect the mental health of their staff, drawing on research from frontline responders.

Specifically, this review aimed to (1) identify and summarise recent early psychological intervention programmes that were administered to prevent or minimise psychological harm in frontline responders, through a systematic review; and (2) assess the potential suitability of implementing these interventions within the healthcare workforce using a healthcare service evaluation framework.

\section{METHODOLOGY}

Our methodology involved (1) a systematic review of early psychological intervention programmes tested in frontline responders; and (2) a healthcare service evaluation framework that reviewed the suitability of each programme for widespread implementation across healthcare workers based on the criteria of effectiveness, content applicability and feasibility of delivery. ${ }^{23}$ Frontline responders are defined here as individuals trained to provide services in emergency or disaster settings, such as healthcare workers and security forces. Early psychological interventions are defined here as programmes designed to prevent or reduce mental health impact with delivery commencing within 3 months of exposure to a traumatic event, following recently revised guidelines from the International Society for Traumatic Stress Studies (ISTSS).$^{24}$ According to the recent ISTSS guidelines, interventions were further classified as universal with single or multiple prevention sessions, selective/ indicated with single or multiple prevention sessions, or early treatment with single or multiple treatment sessions. Universal interventions target all trauma-exposed individuals regardless of risk, selective/indicated interventions target individuals at risk of developing symptoms or with 
early signs of symptoms, and early treatment interventions target individuals after the development of a disorder. ${ }^{24}$

\section{Systematic review}

For the systematic review, a single reviewer searched for early psychological intervention programmes that aim to prevent or reduce mental health issues and that have been tested in frontline responders. The single reviewer examined the identified records twice to minimise error and a second author reviewed the final articles. The search and reporting strategy followed the Preferred Reporting Items for Systematic Reviews and Meta-Analyses guidelines $^{25}$ and was conducted in July 2020 across Embase, Web of Science, PsycINFO, Google Scholar and crossreferencing of reference lists. Using the appropriate search term strategy for each database, the Ovid searches included the following keywords:

(Health care worker* OR healthcare worker* OR health care staff OR healthcare staff OR medical staff OR medical worker* OR frontline worker* OR frontline staff) AND (mental health OR psychological impact OR PTSD OR post-traumatic stress* OR anxiety OR depression) AND (prevent* OR intervention*) AND (covid-19 OR coronavirus OR outbreak* OR epidemic* OR pandemic* OR disaster).
The terms were used as free-text words. Articles were first screened for relevance to the topic by their title and abstract and if they appeared suitable then the full text was downloaded (see figure 1). Risk of bias was assessed at the review process and study levels, according to Risk Of Bias In Systematic reviews guidelines. ${ }^{26}$ Studies were summarised based on their tested population, disaster context, study design, follow-up and outcome measures.

\section{Eligibility criteria}

- Early psychological interventions with delivery commencing within 3 months of the traumatic event, including universal, selective/indicated, or early treatment interventions according to the classification system previously outlined.

- Intervention tested in frontline responders.

- Empirical studies and doctoral theses with a clear theoretical framework that is based on psychological theory.

- Psychological outcome measurements of positive mental health outcomes such as resilience, coping, and life satisfaction and/or negative mental health outcomes such as PTSD, depression and anxiety.

- Longitudinal research design with a baseline and post-treatment follow-up(s).

- Published within the last 15 years.

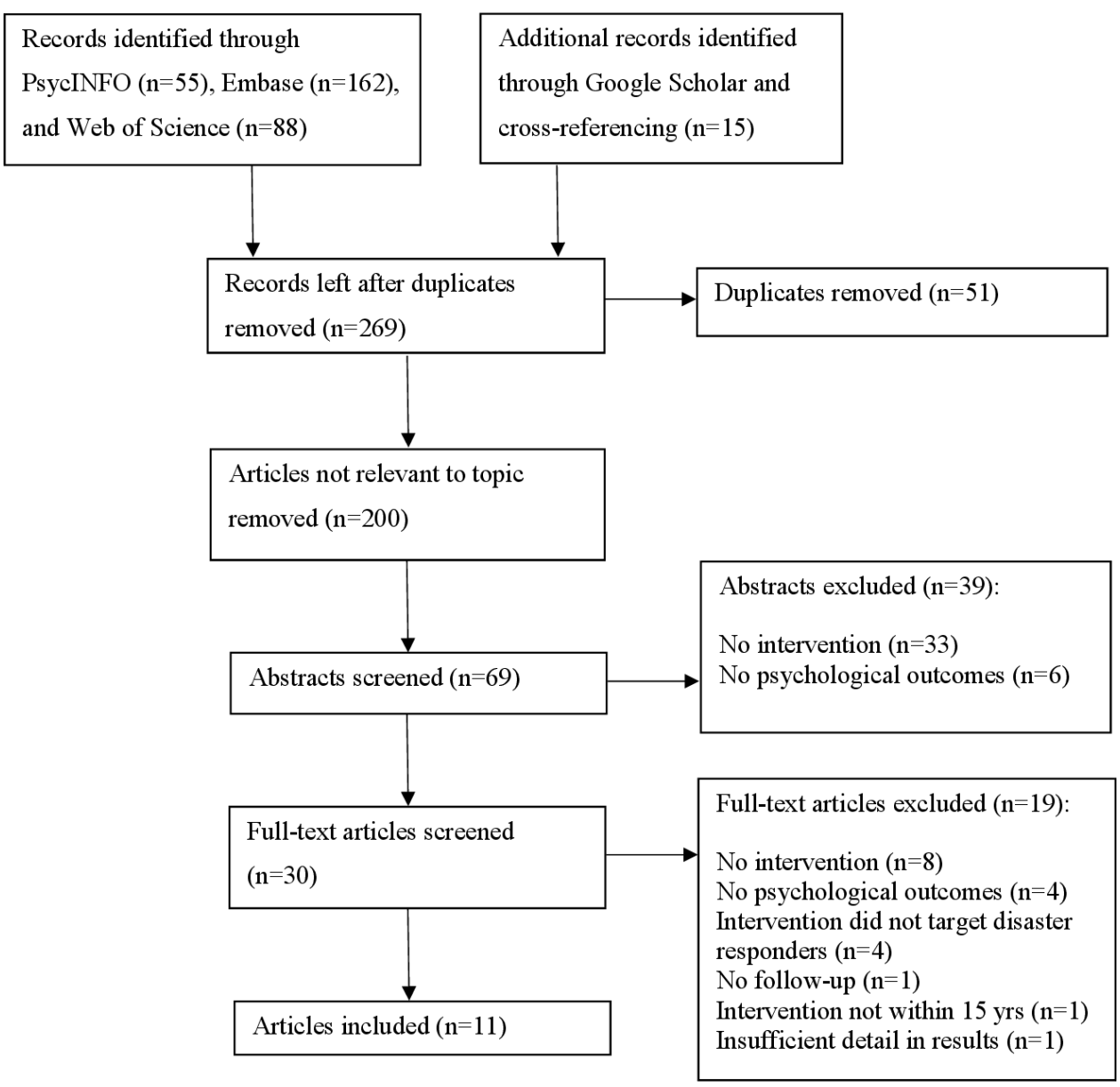

Figure 1 Study flow diagram. 
In contrast, non-psychological (eg, medical, drug and physical) interventions, non-English studies, and purely descriptive, qualitative, or case study designs were excluded. Finally, studies were excluded if the proposed intervention programme is explicitly recommended against by clinical practice guidelines, such as psychological debriefing. ${ }^{27} 28$

\section{Healthcare service evaluation framework}

After intervention programmes were identified through the systematic review, they were rated on their suitability for implementation within healthcare workplaces, based on criteria adapted from an evaluation framework for healthcare programmes. ${ }^{23}$ Each programme was evaluated using three core components of: (1) effectiveness, in this case for reducing psychological distress outcomes or increasing positive psychological outcomes; (2) content applicability to healthcare settings, to determine whether the theoretical content and programme components are relevant for healthcare workers; and (3) feasibility of implementation, including ease of delivery, accessibility and cost.

\section{Patient and public involvement}

There was no patient or public involvement due to the nature of the review.

\section{RESULTS}

\section{Study selection}

The search strategy in the systematic literature review identified 320 potentially relevant articles, including 305 within the databases of PsycINFO, Embase and Web of Science, and an additional 15 articles through manually searching Google Scholar and cross-referencing of reference lists (figure 1). After duplicates were removed and titles and abstracts were screened for relevance, 30 full texts of articles were downloaded. Nineteen studies were excluded in the full-text screening stage. Finally, a total of 12 studies were identified ( 11 articles, see table 1 ). Risk of bias was assessed in the review process and the only risk identified was the absence of a second reviewer. Risk of bias was also assessed at the study level, which showed that the study by Farchi $e t a l^{29}$ did not meet eligibility criteria due to participants not being frontline responders and was therefore excluded.

\section{Summary of study characteristics}

Six psychological intervention programmes were identified across 12 studies (psychological first aid (PFA); eye movement desensitisation and reprocessing (EMDR); anticipate, plan, and deter (APD); resilience at work (RAW); resilience and coping for the healthcare community (RCHC) and trauma risk management (TRiM)). The included programmes were tested in frontline responder populations of medical and paramedic workers, firefighters, community healthcare workers, social service providers, military forces, police officers, forensic workers and other non-professionals trained to respond to emergency or disaster situations (see table 1). Disaster contexts included Ebola, ${ }^{30}$ human massacres, ${ }^{3132}$ military deployment, ${ }^{33}$ car crash fatalities, ${ }^{29}$ maritime collisions ${ }^{34}$ and other various local disasters. ${ }^{34-39}$ There were five randomised controlled trials (RCTs), ${ }^{34-36} 3840$ four crosssectional studies ${ }^{30} 323439$ and three quasi-experimental designs. ${ }^{31} 33$ Follow-up periods ranged from 1 week to 18 months (where specified). PFA, RCHC, APD and RAW were classified as universal, EMDR was classified as selective/indicated in one study and early treatment in the other study, and TRiM was classified as a combination of universal and selective/indicative. PFA and RCHC interventions involved single-treatment sessions; EMDR had single and multiple sessions; and APD, RAW and TRiM had multiple sessions. EMDR and RAW were individuallevel interventions, PFA and RCHC were group-format interventions, and APD and TRiM were delivered across the whole organisation.

The included studies used a variety of measures across the outcome domains of psychological distress (General Health Questionnaire and Depression, Anxiety and Stress Scales-Short Form for psychopathology; Perceived Stress Scale and Stress Arousal Checklist for stress; Impact of Event Scale-Revised, Short Post-Traumatic Stress Disorder Rating Interview, and PTSD Checklist-Civilian for PTSD; Cognitive Fusion Questionnaire for cognitive fusion; Acceptance and Action Questionnaire for experiential avoidance) and positive psychological change (Brief COPE, Ways of Coping, and Coping Self-Efficacy Scale for coping; Connor-Davidson Resilience Scale and Brief Resilience Scale for resilience; Life Orientation Test-Revised for optimism; Freiburg Mindfulness Inventory for mindfulness; Self-Compassion Scale-Short Form for self-compassion; 6-item questionnaire for life satisfaction; Professional Quality of Life for professional quality of life). Additional outcomes included perceived stigma and barriers (Military Stigma Scale and internal and external stigma questionnaire for stigma; Stigma and Barriers to Care Questionnaire for barriers to helpseeking), perceived PFA knowledge (PFA knowledge questionnaire), and occupational functioning (history of minor disciplinary offence rates and absenteeism rates).

\section{Early psychological intervention programmes}

The following section provides a brief description of each early psychological intervention programme included in this review, categorised as universal, selective/indicated, and/or early treatment.

\section{Universal}

PFA is a widely used psychological programme for disaster situations that provides access to emotional, social and physical support. ${ }^{41}$ It aims to reduce short-term and long-term psychological effects of disasters and traumatic events through promoting adaptive functioning and coping. It also offers practical care and immediate support via empathic listening and information on 


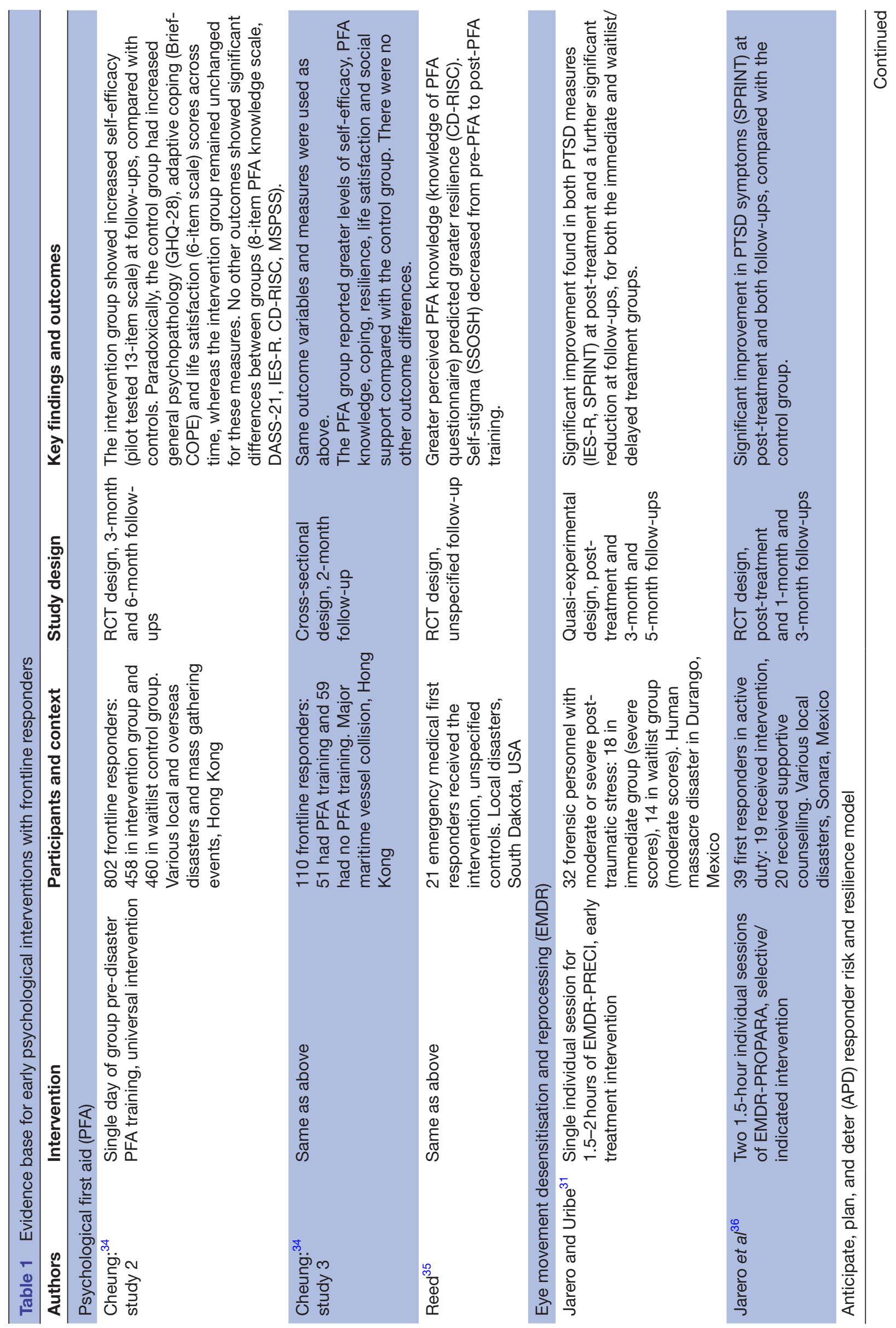



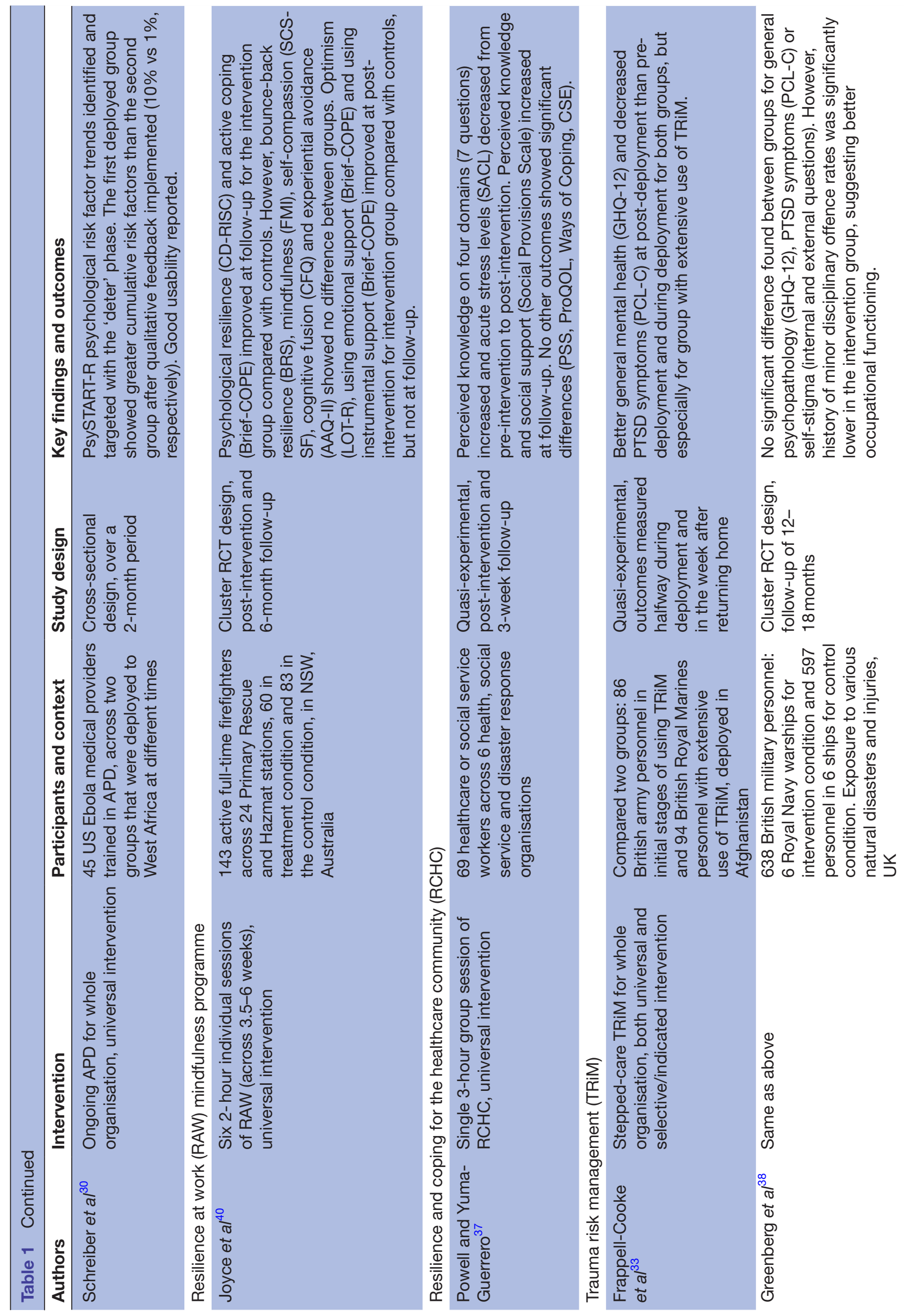

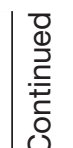




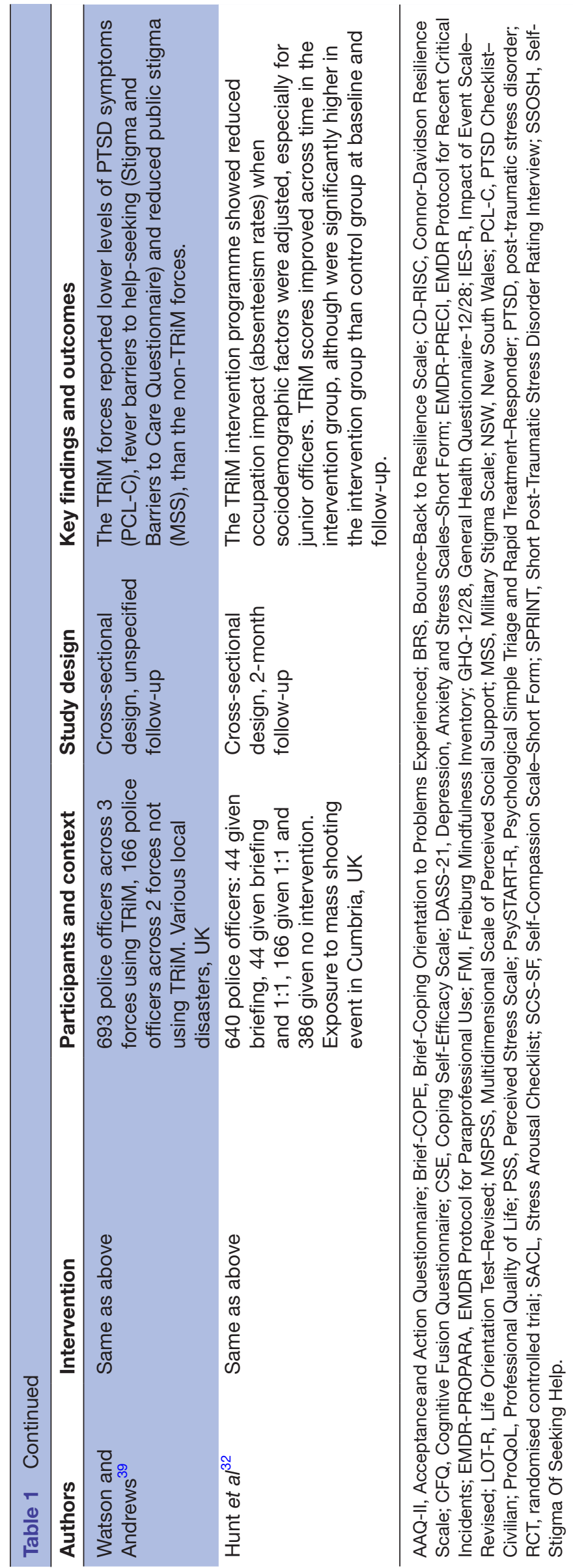


psychosocial services. It is based on the concept of resilience and involves five empirically supported disaster and mass trauma intervention principles, which include promoting a sense of (a) safety, (b) calming, (c) selfefficacy and community-efficacy, (d) connectedness and (e) hope. ${ }^{41}$ It has eight core helping actions and goals that are described in table 2. ${ }^{42}$ PFA can be offered via group or individual format, in a single session.

APD responder risk and resilience model aims to assess and manage psychological risk and resilience in healthcare workers and their families across pre-incident, response and recovery stages of public health emergencies. ${ }^{30}$ Specifically, the APD model consists of a three-step action plan, with the 'anticipate' phase involving preevent stress inoculation training to prepare healthcare personnel for the psychosocial impact of mass trauma events. In the 'plan' phase, staff develop a personal resilience plan, where they can identify and document their expected stress responses and challenges as well as support systems and coping strategies. The final 'deter' phase involves teaching staff how and when to activate their personal resilience plan during stress exposure and encouraging them to use the Psychological Simple Triage and Rapid Treatment-Responder (PsySTART-R) selftriage system. PsySTART-R is a web-based mobile-friendly application and self-assessment tool that tracks daily exposure to traumatic stress, assesses psychological risk factors and gives confidential feedback. The APD model targets the whole organisation over time.

RAW mindfulness programme, renamed recently to Mindarma, is an online intervention designed to increase resilience in high-risk workers, such as first responders. ${ }^{40}$ The RAW is an evidence-based programme that combines principles from mindfulness, CBT, acceptance and commitment therapy, and self-compassion approaches. Specific strategies covered across the programme target mindfulness, resilience, unhelpful thoughts, avoidance, personal values, self-care and compassion. The content is delivered individually via internet format. The original programme runs for 6 sessions of 20-25 min each and the revised current version is 10 sessions of $15 \mathrm{~min}$ each. There is a minimum 3-day break in between sessions to enable skills practice. Sessions include interactive exercises, audio-recordings and animations. Four half-hour group webinars delivered by a senior psychologist are available in conjunction to the programme, to encourage engagement and motivation of users. Moreover, the RAW (or Mindarma) programme is an individual intervention with an optional group component.

RCHC is a recent post-disaster intervention designed to reduce psychological distress and increase resilience in healthcare and social service providers. ${ }^{43}$ In particular, it aims to build healthy coping strategies for dealing with past, current, and future disasters and foster resilience through promoting support between colleagues. It combines psychoeducation and mindfulness practices in an interactive group format that uses solution-focused techniques and action learning theory. There are five core modules: common reactions to stress, how the brain reacts to severe stress and trauma, healthcare provider responses to stress and traumatic events, coping with stress and trauma with individual strategies, and coping with stress and trauma with collective strategies. RCHC is delivered as a single session.

\section{Selective/indicated or early treatment}

EMDR is a psychotherapy treatment designed to alleviate PTSD and other trauma-related symptoms. ${ }^{44}$ EMDR was developed based on the theoretical framework of adaptive information processing, which assumes that trauma symptoms arise from adverse life events being inadequately processed within the brain and stored as traumatic memories that continue to resurface. ${ }^{45}$ In this intervention, a trained EMDR practitioner guides the person to relive their original trauma memory in brief doses while making rapid eye movements, instead of the memory being locked in the nervous system in the form of thoughts, emotions and sensory information about the episode. ${ }^{46}$ EMDR has subprotocols for early intervention in the immediate days and weeks following disaster events, such as the Protocol for Recent Critical Incidents, ${ }^{47}$ Group Traumatic Episode Protocol $^{48}$ and Recent Traumatic Episode Protocol. ${ }^{49}$ It can be delivered in single or multiple sessions, either individually or as a group.

\section{Universal and selective/indicated}

TRiM is a well-established peer-support intervention for first responders that aims to mitigate psychological risk from potentially traumatic events by identifying highrisk individuals and matching them to the appropriate care. ${ }^{50}$ Participation is voluntary and careful consideration is made regarding who it is offered to. Trained management personnel first hold a planning meeting with everyone involved in the potentially traumatic event, to better understand the incident and agree on a tailored response. In the next stage, TRiM practitioners run basic psychoeducational briefings to cover the specific incident as well as how to manage responses. Lastly, high-risk individuals are asked to take part in a semistructured risk assessment interview and are linked with appropriate psychological support. Essentially, TRiM provides a framework for organisations to monitor and effectively manage the psychological impact of potentially traumatic events in their employees, through a whole organisation stepped-care intervention.

\section{Healthcare service evaluation framework}

The following section evaluates each programme within the healthcare workforce, based on the criteria of effectiveness, content applicability and feasibility with this population. A snapshot summary of the objective, content, endorsements, effectiveness, feasibility and overall suitability for healthcare settings is summarised in table 2. 

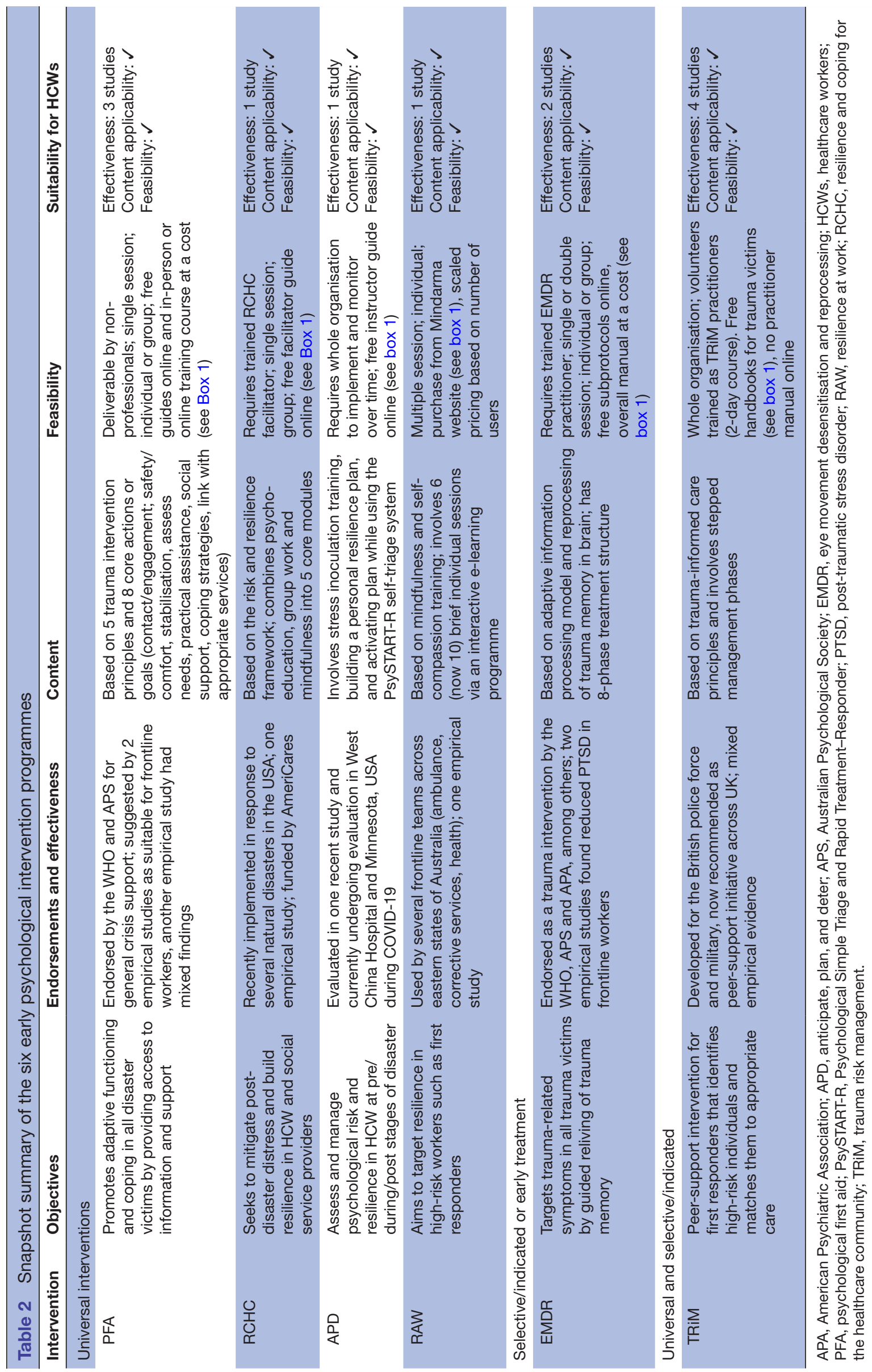

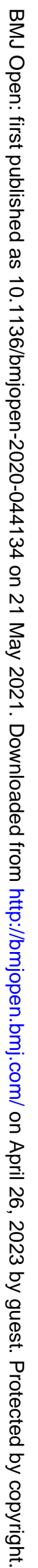




\section{Psychological first aid}

\section{Effectiveness}

Despite being one of the most widely known and used programmes in the general community ${ }^{51}$ as well as endorsed by the $\mathrm{WHO}^{52}$ and Australian Psychological Society ${ }^{53}$ PFA has limited empirical evidence. ${ }^{54}$ To our knowledge, there has been no empirical study in which frontline workers were the direct recipients of PFA after a trauma event. However, three studies reported on the mental health benefits to frontline workers after receiving a single day of training in PFA delivery (see table 2). Psychological benefits were reported in medical staff $^{35}$ and other professional and non-professional first responders. ${ }^{34}$ These benefits included improvements in positive psychological outcomes of resilience, self-efficacy, perceived knowledge and social support. Reduced perceived self-stigma was also found. There was no evidence for change in general psychopathology, coping or life satisfaction in the intervention group, although controls showed increased general psychopathology over time. The authors suggest that PFA brings psychological benefits for the person delivering it, in addition to their recipients, as it teaches coping strategies and facilitates connection with support systems and services, which can be used to protect the self as well as support others.

\section{Content applicability}

PFA is a generic disaster relief approach that can be implemented either during or immediately after the disaster and can apply to anyone impacted by the event. Its broadspectrum response strategy makes it easily generalisable to multiple population groups and settings with a variety of psychological needs. It allows for local adaptations, thus is suitable for healthcare workers.

\section{Feasibility}

PFA training typically runs for a single day. Specialised mental health practitioners are not required to deliver PFA, although it assumes that trainees have basic knowledge in helping distressed individuals. Short training courses in delivering PFA are available and costs are variable, but can be minimised through group training, train the trainee schemes and online training (see box 1).

\section{Eye movement desensitisation and reprocessing Effectiveness}

Despite continuous scepticism from the scientific community, ${ }^{55}$ EMDR is steadily becoming a popular and well-established intervention for treating trauma-related symptoms. ${ }^{56}$ It is universally endorsed by the WHO, ${ }^{57}$ among others. ${ }^{275859}$ Reduced PTSD symptoms have been found across forensic personnel and first responders receiving EMDR. ${ }^{31}{ }^{36}$ Given that frontline workers across healthcare, forensic and first responder populations are frequently exposed to traumatic events and are prone to experience secondary traumatisation, ${ }^{4}$ EMDR may also be applicable for treating trauma-related symptoms in healthcare providers.
Box 1 Links to more information on each included programme

Psychological first aid (PFA)

- There are several free PFA guides available online. The $\mathrm{WHO}^{52}$ pro-

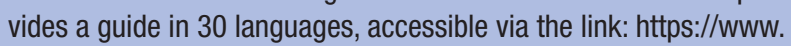
who.int/mental_health/publications/guide_field_workers/en/.

- The Australian Red Cross provides another PFA guide endorsed by the Australian Psychological Society, ${ }^{53}$ accessible via the link: https://www.redcross.org.au/getmedia/dc21542f-16e4-44ba8e3a-4f6b907bba6f/Psychological-First-Aid-An-AustralianGuide-04-20.pdf.aspx.

Eye movement desensitisation and reprocessing (EMDR)

- A comprehensive practice manual by Marilyn Luber ${ }^{71}$ can be purchased online that contains models, scripted protocols and summary sheets for early EMDR intervention.

- An overview and protocol manual is available for free for EMDR Recent Traumatic Episode Protocol ${ }^{49}$ : https://emdrresearchfoundation.org/toolkit/rtep-manual.pdf; for EMDR-Protocol for Recent Critical Incidents ${ }^{72}$ : https://emdrresearchfoundation.org/toolkit/preci.pdf; and for EMDR Group Traumatic Episode Protocol ${ }^{73}$ : https:// emdrresearchfoundation.org/toolkit/gtep.pdf.

The anticipate, plan, and deter (APD) responder risk and resilience model

- An instructor guide for the APD model with relevant worksheets ${ }^{74}$ is freely available online: http://file.lacounty.gov/SDSInter/ dhs/221064_AnticipatePlanandDeterlnstructorManual-FINAL.pdf.

\section{Resilience at work (RAW) mindfulness programme}

- The RAW (now called Mindarma) programme can be purchased via the website below, ${ }^{75}$ which contains information including pricing, media, workshops and contact details: https://www.mindarma.com/ home/.

- More information can also be found on the Black Dog Institute website $^{76}$ : https://www.blackdoginstitute.org.au/education-services/ workplaces/workplace-programs/mindarma/.

\section{Resilience and coping for the healthcare community} (RCHC)

- While the full RCHC manual is not available online, the author can be contacted at: paula.yuma@colostate.edu and more information on each module ${ }^{43}$ can be found at: https://digitalcommons.library.tmc. edu/jfs/vol19/iss1/8/.

\section{Trauma risk management (TRiM)}

- Several TRiM handbooks designed for trauma victims are available online. For more information on TRiM training, see ${ }^{77}$ : http://www. marchonstress.com/page/p/trim_faqs.

- TRiM handbook by the British Royal Navy ${ }^{78}$ : http://c69011.r11.cf3. rackcdn.com/d951c5627eb44b3789e84292d1e2c1fa-0x0.pdf.

- TRiM handbook by the UK Hampshire Fire and Rescue Service and Hampshire Constabulary ${ }^{79}$ : https://documents.hants.gov.uk/corprhantsweb/Traumahandbook.pdf.

\section{Content applicability}

The Protocol for Paraprofessional Use in acute trauma situations (PROPARA) was developed as an early EMDR intervention for first responders. ${ }^{36}$ Like other early intervention models, it follows the same eight-phase structure as the standard treatment protocol, ${ }^{60}{ }^{61}$ yet differs in the specific processing techniques used and how the 
traumatic episode is conceptualised. ${ }^{29} 32$ PROPARA would need no further adaption for use with healthcare workers who experience trauma or disaster events. Other early EMDR protocols may also potentially be adapted to support the needs of the healthcare workforce.

\section{Feasibility}

Early EMDR intervention typically lasts for a couple of hours, whereas the standard intervention spans across several days. An EMDR practitioner with specialist training must deliver EMDR therapy to ensure correct delivery, making it a costly treatment. There are several early EMDR intervention protocols available online (see box 1 ).

\section{APD responder risk and resilience model \\ Effectiveness}

There is limited evaluation of the APD model in the literature. In one recent study, however, the full APD model was implemented in medical providers during the Ebola crisis, who showed reduced psychological risk factors across time. ${ }^{30}$ The APD model is part of a two-stage psychological rehabilitation plan currently underway in West China Hospital, which seeks to prevent further psychological impact of COVID-19 on healthcare workers. It is also part of a larger psychological intervention study for healthcare organisations to provide to their staff during the COVID-19 pandemic, currently undergoing evaluation in Minnesota. ${ }^{62}$ While research on the APD model is in its infancy, it has shown promising effectiveness in healthcare workers.

\section{Content applicability}

The APD model was explicitly intended as a psychological intervention for healthcare workers, with theoretical frameworks of risk and resilience tailored to suit this population. Therefore, its content is already applicable to healthcare settings.

\section{Feasibility}

The APD risk and resilience model requires the organisation to implement the system throughout all stages of disaster response and regularly monitor risk. Therefore, it is relatively resource-intensive compared with other programmes, as it requires continuous management over time (see box 1 for a full instructor guide).

\section{RAW mindfulness programme \\ Effectiveness}

The RAW programme shows promising preliminary results, with a recent empirical study finding improved positive mental health outcomes of resilience and active coping in a group of full-time firefighters at post-treatment. ${ }^{40}$ This study also provided evidence for increased optimism and use of support, but no evidence for change in resilience, mindfulness, self-compassion, cognitive fusion or experiential avoidance. The programme, now called Mindarma and adapted to run for 10 sessions, has been adopted by several frontline organisations in the eastern states of Australia including ambulance, corrective and health services. The programme is also fully endorsed by the Black Dog Institute-a not-for-profit organisation and world leader in mental health services and research.

\section{Content applicability}

The RAW programme was designed to protect the wellbeing of high-risk workers through resilience training. The programme can be tailored to the unique needs of the workforce, through customisation of content such as scripts, animations and programming. Therefore, it appears suitable for healthcare professionals in high-risk workplaces.

\section{Feasibility}

The online RAW (or Mindarma) programme can be implemented quickly and easily at a low cost by contacting the owners of the programme via their website (see box 1). Pricing is scaled depending on the number of users in the organisation and organisation managers can receive in-person training workshops to ensure they are well informed.

\section{Resilience and coping for the healthcare community Effectiveness}

The RCHC intervention has demonstrated some effectiveness at reducing psychological impact in healthcare workers, producing positive psychological outcomes of increased perceived knowledge and social support and decreased acute stress levels in a single study. ${ }^{37} \mathrm{RCHC}$ was recently implemented in several other areas also affected by natural disasters in the USA, such as typhoon-affected Saipan in 2015 and flood-affected Shreveport in 2017, and is currently undergoing evaluation as a recovery response strategy for Hurricane Harvey in Texas and Hurricane Maria in Puerto Rico, funded by AmeriCares. ${ }^{43}$

\section{Content applicability}

The RCHC uses a risk and resilience framework that has been carefully adapted for use with healthcare and social service providers by acknowledging the high-risk exposure of this workforce and the incorporation of appropriate strategies to build resilience. ${ }^{43}$ RCHC contains suitable content for healthcare workers, as it was explicitly designed for this population.

\section{Feasibility}

The RCHC can be delivered to staff in 3 hours and a trained RCHC facilitator is required to deliver the intervention. There is no full manual available online (see box 1).

\section{Trauma risk management \\ Effectiveness}

The TRiM programme has mixed findings, with two studies showing reduced psychological impact in police and Army officers and one study showing no change in military officers. ${ }^{33} 383963$ However, there is evidence to suggest it can reduce stigma and barriers to help-seeking 
as well as improve occupational functioning in these populations. ${ }^{32} 3839$

\section{Content applicability}

Initially developed in the British military and used within police officers, TRiM is now used by many different organisations across the UK. It offers an evidence-based framework for early indication of who may go on to develop mental health symptoms after a traumatic event and how this should be managed to ensure the best conditions for psychological recovery. This framework is generic enough that it is also suitable for use in healthcare settings. The overlap in traumatic workplace experiences between healthcare and other frontline workers suggests that the TRiM programme would show similar results in healthcare settings.

\section{Feasibility}

The TRiM programme is intended to be delivered by volunteer personnel within the organisation, ideally from a managerial position. TRiM practitioner courses typically run over 2 or more days. Several TRiM handbooks designed for trauma victims are available online (see box 1).

\section{DISCUSSION}

The key objective of this paper was to equip healthcare service providers with practical information on how to protect the mental health of healthcare professionals during local and global disasters. Since the evidence base for early psychological interventions within healthcare workers is limited and personnel on the frontline also face exposure to traumatic events, all frontline responders were considered in this review. Evidence was searched for early psychological interventions designed to prevent or reduce psychological harm and that were tested in frontline workers during recent disease outbreaks and disasters. Each included programme was described and evaluated based on its suitability for rapid implementation among the healthcare workforce using the criteria of effectiveness, content applicability and feasibility. This evaluation framework seeks to provide a current workplace mental health response guide for healthcare service providers.

Generally, the evidence base was limited across all intervention programmes. Six early psychological programmes were identified across 12 studies, with four programmes classified as universal, one classified as selective/indicated in one study and early treatment in another study, and one classified as both universal and selective/indicated, due to its stepped-care approach. ${ }^{24}$ Out of the sparse number of studies, PFA, EMDR and TRiM were tested in frontline responders across at least two studies each, in addition to being applicable and feasible for rapid implementation within the healthcare workforce (see table 2). In particular, these interventions demonstrated improved psychological outcomes at follow-ups across two or more
Box 2 Recommendations for single-session prevention interventions

- Intervention with emerging evidence-psychological first aid.

- Intervention with insufficient evidence-resilience and coping for the healthcare community.

Emerging evidence=two or more studies; insufficient evidence=only one study. Recommendations based on recent International Society for Traumatic Stress Studies guidelines. ${ }^{24}$

studies, with several increased positive psychological outcomes found with PFA, ${ }^{34} 35$ several reduced negative psychological outcomes found with TRiM ${ }^{32} 3339$ and reduced PTSD levels found with EMDR. ${ }^{31}{ }^{36}$ However, TRiM requires further research, due to inconsistent findings of effectiveness across various studies with frontline responders. ${ }^{63}$ It is also important to note that the evidence base for PFA involves frontline workers receiving training in PFA delivery rather than as direct recipients of the intervention.

The APD, RAW (or Mindarma) and RCHC programmes are relatively new psychological interventions that have shown promising outcomes with frontline workers and appear to be potentially suitable interventions for rapid implementation among healthcare workers. ${ }^{30} 3740$ APD and RCHC were specifically intended for healthcare workers and RAW for high-risk workers, thus these programmes have been tailored to suit this population and may be more appropriate than more generic approaches such as PFA and EMDR. Nevertheless, evidence for APD, RCHC and RAW programmes is limited to a single study each and only the RAW study included a control condition, thus further evaluation of these programmes is required in order to strengthen their evidence base. Boxes 2-4 provide a summary of recommendations for evidence according to the ISTSS guidelines for prevention (ie, universal and selective/indicated) interventions and early treatment programmes with single or multiple sessions. ${ }^{24}$ These tables indicate emerging evidence for PFA and TRiM and insufficient evidence for EMDR, APD, RCHC and RAW, further suggesting the need for additional research on each intervention.

Several promising early psychological interventions did not meet eligibility requirements for this review, yet deserve mention. First, immediate cognitive-functional

Box 3 Recommendations for multiple session prevention interventions

- Intervention with emerging evidence-trauma risk management.

- Intervention with insufficient evidence-eye movement desensitisation and reprocessing; anticipate, plan, and deter; resilience at work.

Emerging evidence=two or more studies; insufficient evidence=only one study. Recommendations based on recent International Society for Traumatic Stress Studies guidelines. ${ }^{24}$ 


\section{Box 4 Recommendations for early treatment}

Intervention with insufficient evidence-eye movement desensitisation and reprocessing.

Insufficient evidence $=$ only one study. Recommendations based on recent International Society for Traumatic Stress Studies guidelines. ${ }^{24}$

PFA (ICF-PFA) was recently proposed as an improved, structured and more immediate disaster relief approach to PFA that targets symptoms of the acute stress reaction by drawing on psychological theories of stress and resilience. ${ }^{29}$ ICF-PFA is recognised as the national PFA model by the Israeli Ministry of Health and has been adopted by several frontline sectors, with the Israeli Defence Forces currently investigating its impact on frontline soldiers. However, this intervention was excluded as it has only been empirically tested in trauma-exposed adolescent students and requires formal evaluation in frontline staff. In addition, a computer-assisted resilience training programme given to Canadian healthcare workers during the SARS outbreak found positive psychological impact at post-treatment, but was excluded from this review due to lack of a follow-up measurement. ${ }^{64} \mathrm{~A}$ recent digital learning package targeting psychological well-being showed good user satisfaction of healthcare workers across the UK during COVID-19 but was also excluded as it used qualitative analysis only. ${ }^{65}$ A study testing a stress management and resilience training programme in medical physicians was also excluded due to absence of disaster situations. ${ }^{66}$ Finally, study protocol is available for an 8-week online CBT programme designed for healthcare workers that is currently undergoing evaluation in the context of COVID-19 in France. ${ }^{67}$ Future research should explore these potential alternative interventions, in addition to those included in this review.

Empirical research on the effectiveness of early psychological interventions for preventing or reducing post-traumatic and other mental health symptoms in healthcare and other frontline workers is limited, with few RCTs available and most interventions targeting community disaster victims. One alternative is to compare the findings of this paper with literature on early psychological interventions tested in other trauma-exposed populations. Recent systematic reviews suggest that early EMDR and trauma-focused CBT are among the most effective individualised programmes for targeting trauma-related symptoms, ${ }^{68} 69$ yet more evidence is still needed on these interventions. ${ }^{70}$ PFA is also recommended as an early intervention for trauma victims, ${ }^{51}$ despite its lack of evidence in comparison with the other interventions. ${ }^{54}$ This wider literature is consistent with the finding that PFA and EMDR are the most evidence-based early psychological interventions for frontline workers to date. To the best of our knowledge, there is currently no research on trauma-focused CBT for frontline responder populations. Nevertheless, it is recommended that anyone with severe or persistent trauma-related symptoms should seek out more intensive and longer term individualised support, such as trauma-focused CBT. ${ }^{11} 1718$

It is possible that several other suitable early psychological interventions exist for frontline workers, in addition to those mentioned above, that have not yet received formal evaluation. This is understandable given the chaotic nature of healthcare services when dealing with mass traumas and disasters. A potential limitation of this paper is that, due to the rapid need for a review in this area, only three databases were searched and a single reviewer assessed the articles. Even though the databases were chosen for their comprehensive coverage of medical and psychological research, it is possible that relevant studies were missed. Additionally, the ongoing character of the COVID-19 pandemic may induce longer periods of elevated traumatic stress in frontline workers compared with the acute trauma from local disasters and accidents, differentiating this context from previous disaster situations. Despite limitations, available past research with all frontline workers can still guide healthcare services in implementing early intervention programmes that might proactively address the mental health fallout of the current COVID-19 crisis, as well as future pandemics and other mass health and trauma crises. This review has outlined several early interventions tested in frontline workers during disease outbreaks and other disasters, which also appear suitable for widespread implementation in the healthcare workforce.

While the process and prioritisation of research can be challenging in the context of mass trauma events, this is an essential area of development. Healthcare systems play a crucial role in evaluating the interventions they implement, in order to build the much needed evidence base for preventing and reducing psychological impact in healthcare workers and elucidating best practices for services in managing future disasters. Healthcare services are typically vigilant to addressing the physical safety of staff in the workplace and the psychological safety of staff must also be given equal priority. Indeed, healthcare services have a duty of care to equip their staff with support and psychological skills to assist with the mental health challenges they will inevitably face as part of their courageous frontline work they do for the benefit of the broader community, particularly during times of mass crises. This review of the evidence for early psychological interventions within frontline staff and the consideration of suitability for healthcare settings is intended to be a helpful resource to guide healthcare and other frontline services seeking to select an intervention to suit the needs of their organisation and its employees.

Contributors JJH, LS, TH and FW contributed to manuscript drafts and selection criteria. JJH conducted data extraction with review from FW. FW also provided expertise on healthcare service implementation and research. LS provided expertise on clinical programme implementation and informed care. TH contributed to the section on debriefing.

Funding This work was supported by an RTP scholarship from the University of Western Australia (grant number BU0014010306107). 
Competing interests None declared.

Patient consent for publication Not required.

Ethics approval Ethics approval was not required as the methodology comprised a literature review and there was no testing of participants. Informed consent was not applicable.

Provenance and peer review Not commissioned; externally peer reviewed.

Data availability statement Data sharing not applicable as no datasets generated and/or analysed for this study.

Open access This is an open access article distributed in accordance with the Creative Commons Attribution Non Commercial (CC BY-NC 4.0) license, which permits others to distribute, remix, adapt, build upon this work non-commercially, and license their derivative works on different terms, provided the original work is properly cited, appropriate credit is given, any changes made indicated, and the use is non-commercial. See: http://creativecommons.org/licenses/by-nc/4.0/.

ORCID iD

Jasmine Jean Hooper http://orcid.org/0000-0003-2134-1085

\section{REFERENCES}

1 Kleim B, Westphal M. Mental health in first responders: a review and recommendation for prevention and intervention strategies. Traumatology 2011;17:17-24.

2 Mao X, Fung OWM, Hu X, et al. Psychological impacts of disaster on rescue workers: a review of the literature. International Journal of Disaster Risk Reduction 2018;27:602-17.

3 Substance Abuse and Mental Health Services Administration. National survey on drug use and health: methodological summary and definitions. 2019. Rockville, MD: Center for Behavioral Health Statistics and Quality, Substance Abuse and Mental Health Services Administration, 2018. https://www.samhsa.gov/data/

4 Arpacioglu S, Gurler M, Cakiroglu S. Secondary traumatization outcomes and associated factors among the health care workers exposed to the COVID-19. Int J Soc Psychiatry 2020:002076402094074.

5 Ray SL, Wong C, White D. Work life conditions, and burnout among frontline mental health care professionals. Traumatology 2013;19:255-67.

6 Joyce S, Tan L, Shand F, et al. Can resilience be measured and used to predict mental health Symptomology among first responders exposed to repeated trauma? J Occup Environ Med 2019;61:285-92

7 Meewisse M-L, Olff M, Kleber R, et al. The course of mental health disorders after a disaster: predictors and comorbidity. J Trauma Stress 2011;24:405-13.

8 Haugen PT, Evces M, Weiss DS. Treating posttraumatic stress disorder in first responders: a systematic review. Clin Psychol Rev 2012;32:370-80.

9 World Health Organization. Mental health and psychosocial considerations during the COVID-19 outbreak, 2020. Available: https://www.who.int/publications-detail-redirect/WHO-2019-nCoVMentalHealth-2020.1

10 Sim MR. The COVID-19 pandemic: major risks to healthcare and other workers on the front line. Occup Environ Med 2020;77:281-2.

11 Benhamou K, Piedra A. CBT-Informed interventions for essential workers during the COVID-19 pandemic. J Contemp Psychother 2020;50:275-83.

12 Lai J, Ma S, Wang Y, et al. Factors associated with mental health outcomes among health care workers exposed to coronavirus disease 2019. JAMA Netw Open 2020;3:e203976.

13 Chew NWS C, GKH L, BYQ T. A multinational, multicentre study on the psychological outcomes and associated physical symptoms amongst healthcare workers during COVID-19 outbreak. Brain Behav Immun 2020;1;88:559-65.

14 Du J, Dong L, Wang T, et al. Psychological symptoms among frontline healthcare workers during COVID-19 outbreak in Wuhan. Gen Hosp Psychiatry 2020;67:144-5.

15 Choi KR, Heilemann MV, Fauer A, et al. A second pandemic: mental health spillover from the novel coronavirus (COVID-19). J Am Psychiatr Nurses Assoc 2020;26:340-3.

16 Kisely S, Warren N, McMahon L. Occurrence, prevention, and management of the psychological effects of emerging virus outbreaks on healthcare workers: rapid review and meta-analysis. BMJ 2020;5;369:m1642.
17 Forbes D, Creamer M, Phelps A, et al. Australian guidelines for the treatment of adults with acute stress disorder and post-traumatic stress disorder. Aust N Z J Psychiatry 2007;41:637-48.

18 Billings J, Greene T, Kember T, et al. Supporting hospital staff during COVID-19: early interventions. Occup Med 2020;70:327-9.

19 Clement S, Schauman O, Graham T, et al. What is the impact of mental health-related stigma on help-seeking? A systematic review of quantitative and qualitative studies. Psychol Med 2015;45:11-27.

20 World Health Organization. Mental health in emergencies, 2019. Available: https://www.who.int/news-room/fact-sheets/detail/mentalhealth-in-emergencies

21 Brooks SK, Rubin GJ, Greenberg N. Traumatic stress within disasterexposed occupations: overview of the literature and suggestions for the management of traumatic stress in the workplace. Br Med Bull 2019;129:25-34.

22 Roberts NP, Kitchiner NJ, Kenardy J, et al. Systematic review and meta-analysis of multiple-session early interventions following traumatic events. Am J Psychiatry 2009;166:293-301.

23 Reeve C, Humphreys J, Wakerman J. A comprehensive health service evaluation and monitoring framework. Eval Program Plann 2015;53:91-8.

24 O'Donnell ML, Pacella BJ, Bryant RA. Chapter 8: early intervention for trauma-related psychopathology. In: Forbes D, Bisson JI, Monson $\mathrm{CM}$, et al, eds. Effective treatments for PTSD: practice guidelines from the International Society for traumatic stress studies. 3rd ed. New York: Guildford Publications, 2020: 117-36.

25 Moher D, Liberati A, Tetzlaff J, et al. Preferred reporting items for systematic reviews and meta-analyses: the PRISMA statement. PLoS Med 2009;6:e1000097.

26 Whiting P, Savović J, Higgins JPT, et al. ROBIS: a new tool to assess risk of bias in systematic reviews was developed. J Clin Epidemiol 2016;69:225-34.

27 National Institute for Health and Care Excellence (NICE). PostTraumatic stress disorder, 2018. Available: https://www.nice.org. uk/guidance/ng116/resources/posttraumatic-stress-disorder-pdf66141601777861

28 Phoenix Australia - Centre for Posttraumatic Mental Health. Australian guidelines for the treatment of acute stress disorder and posttraumatic stress disorder. Melbourne, Victoria: Phoenix Australia, 2013. https://www.phoenixaustralia.org/wp-content/uploads/2015/ 03/Phoenix-ASD-PTSD-Guidelines.pdf

29 Farchi M, Hirsch-Gornemann MB, Whiteson A, et al. The six Cs model for immediate cognitive psychological first aid: from helplessness to active efficient coping. Int J Emerg Ment Health 2018;20:1-12.

30 Schreiber M, Cates DS, Formanski S. Maximizing the resilience of healthcare workers in Multi-hazard events: lessons from the 2014-2015 Ebola response in Africa. Mil Med 2019;1;184:114-20.

31 Jarero I, Uribe S. The EMDR protocol for recent critical incidents: follow-up report of an application in a human massacre situation. $J$ EMDR Prac Res 2012;6:50-61.

32 Hunt E, Jones N, Hastings V, et al. Trim: an organizational response to traumatic events in Cumbria Constabulary. Occup Med 2013;63:549-55.

33 Frappell-Cooke W, Gulina M, Green K, et al. Does trauma risk management reduce psychological distress in deployed troops? Occup Med 2010;60:645-50.

34 Cheung YL. Psychological first aid as a public health disaster response preparedness strategy for responders in critical incidents and disasters [dissertation on the Internet]. Hong Kong: The Chinese University of Hong Kong, 2014. https://search.proquest.com/ docview/1691127433/abstract/F18583B127484126PQ/1

35 Reed AJ. Community-based psychological first aid for emergency medical service providers [dissertation on the Internet]. South Dakota: University of South Dakota, 2013. https://search.proquest. com/docview/1658771774/abstract/791C1BC615484D7CPQ/1. (cited 2020 Aug 22).

36 Jarero I, Amaya C, Givaudan M, et al. EMDR individual protocol for Paraprofessional use: a randomized controlled trial with first responders. J EMDR Prac Res 2013;7:55-64.

37 Powell T, Yuma-Guerrero P. Supporting community health workers after a disaster: findings from a mixed-methods pilot evaluation study of a psychoeducational intervention. Disaster Med Public Health Prep 2016;10:754-61.

38 Greenberg N, Langston V, Everitt B, et al. A cluster randomized controlled trial to determine the efficacy of trauma risk management (TRIM) in a military population. J Trauma Stress 2010;23:430-6.

39 Watson L, Andrews L. The effect of a trauma risk management (TRIM) program on stigma and barriers to help-seeking in the police. Int J Stress Manag 2018;25:348-56. 
40 Joyce S, Shand F, Lal TJ, et al. Resilience@work mindfulness program: results from a cluster randomized controlled trial with first responders. J Med Internet Res 2019;21:e12894.

41 Hobfoll SE, Watson P, Bell CC. Five essential elements of immediate and Mid-Term mass trauma intervention: empirical evidence. Psychiatry Interpers Biol Process 2007;1;70:283-315.

42 Ruzek JI, Brymer MJ, Jacobs AK, et al. Psychological first aid. J Ment Health Couns 2007;29:17-49.

43 Yuma P, Powell T, Scott J. Resilience and coping for the healthcare community: a post-disaster group work intervention for healthcare and social service providers. J Fam Strengths 2019;19:8.

44 Shapiro F. EMDR: basic principles, protocols, and procedures. NY, New York: Guilford Press, 1995.

45 Felitti VJ, Anda RF, Nordenberg D. Relationship of childhood abuse and household dysfunction to many of the leading causes of death in adults: the adverse childhood experiences (ACE) study. Am J Prev Med 1998;1;14:245-58.

46 Van der Kolk BA. The assessment and treatment of complex PTSD. In: Yehuda R, ed. Treating trauma survivors with PTSD. Washington, DC: American Psychiatric Press, Inc, 2008: 127-56.

47 Jarero I, Artigas L, Luber M. The EMDR protocol for recent critical incidents: application in a disaster mental health continuum of care context. J EMDR Prac Res 2011;5:82-94.

48 Lehnung M, Shapiro E, Schreiber M, et al. Evaluating the EMDR group traumatic episode protocol with refugees: a field study. $J$ EMDR Prac Res 2017;11:129-38.

49 Shapiro E, Laub B. The recent traumatic episode protocol (R-TEP): an integrative protocol for early EMDR intervention (EEI). New York, NY: Springer Publishing, 2014.

50 Greenberg N. TRiM-Trauma risk management: an organisational approach to personnel management in the wake of traumatic events. Shrivenham, UK: Internal Royal Naval Publication, Joint Stress Management Training Centre, 2006.

51 Fox JH, Burkle FM, Bass J, et al. The effectiveness of psychological first aid as a disaster intervention tool: research analysis of peerreviewed literature from 1990-2010. Disaster Med Public Health Prep 2012;6:247-52.

52 Snider L, Van Ommeren M, Schafer A. Psychological first aid: guide for field workers. Geneva, Switzerland: World Health Organization, War Trauma Foundation and World Vision International, 2011.

53 Australian Psychological Society, Australian Red Cross. Psychological first aid: supporting people affected by disaster in Australia. Victoria, Australia: Author, 2020. https://www.redcross. org.au/getmedia/dc21542f-16e4-44ba-8e3a-4f6b907bba6f/ Psychological-First-Aid-An-Australian-Guide-04-20.pdf.aspx

54 Dieltjens T, Moonens I, Van Praet K, Praet KV, et al. A systematic literature search on psychological first aid: lack of evidence to develop guidelines. PLoS One 2014;9:e114714.

55 Russell MC. Scientific resistance to research, training and utilization of eye movement desensitization and reprocessing (EMDR) therapy in treating post-war disorders. Soc Sci Med 2008;67:1737-46.

56 Wilson G, Farrell D, Barron I, et al. The use of eye-movement desensitization reprocessing (EMDR) therapy in treating posttraumatic stress Disorder-A systematic narrative review. Front Psychol 2018;9:923.

57 World Health Organization. Guidelines for the management of conditions specifically related to stress. Geneva, Switzerland: Author, 2013. http://www.ncbi.nlm.nih.gov/books/NBK159725/

58 Ursano RJ, Bell B, Eth S. Practice guideline for the treatment of patients with acute stress disorder and posttraumatic stress disorder. Arlington, VA: American Psychiatric Association, 2004.

59 Australian Psychological Society. Evidence-Based psychological interventions in the treatment of mental disorders: a literature review. Flinders Lane, VIC: Australian Psychological Society, 2018.
60 Shapiro E, Laub B, Intervention EE. Early EMDR intervention (EEI): a summary, a theoretical model, and the recent traumatic episode protocol (R-TEP). J EMDR Prac Res 2008;2:79-96.

61 Shapiro F, Maxfield L. Eye movement desensitization and reprocessing (EMDR): information processing in the treatment of trauma. J Clin Psychol 2002;58:933-46.

62 Albott CS, Wozniak JR, McGlinch BP, et al. Battle Buddies: rapid deployment of a psychological resilience intervention for health care workers during the COVID-19 pandemic. Anesth Analg 2020;131:43-54.

63 Whybrow D, Jones N, Greenberg N. Promoting organizational wellbeing: a comprehensive review of trauma risk management: table 1. Occup Med 2015;65:331-6.

64 Maunder RG, Lancee WJ, Mae R, et al. Computer-Assisted resilience training to prepare healthcare workers for pandemic influenza: a randomized trial of the optimal dose of training. BMC Health Serv Res 2010;10:72.

65 Blake H, Bermingham F, Johnson G, et al. Mitigating the psychological impact of COVID-19 on healthcare workers: a digital learning package. Int J Environ Res Public Health 2020;17. doi:10.3390/ijerph17092997. [Epub ahead of print: 2604 2020].

66 Sood A, Prasad K, Schroeder D, et al. Stress management and resilience training among department of medicine faculty: a pilot randomized clinical trial. J Gen Intern Med 2011;26:858-61.

67 Weiner L, Berna F, Nourry N, et al. Efficacy of an online cognitive behavioral therapy program developed for healthcare workers during the COVID-19 pandemic: the reduction of stress (REST) study protocol for a randomized controlled trial. Trials 2020;21:870.

68 Roberts NP, Kitchiner NJ, Kenardy J, et al. Early psychological intervention following recent trauma: a systematic review and metaanalysis. Eur J Psychotraumatol 2019;10:1695486.

69 Roberts N, Kitchiner N, Kenardy J. Early psychological interventions to treat acute stress symptoms. Cochrane Database Syst Rev 2010;3:CD007944.

70 Roberts NP, Kitchiner NJ, Kenardy J. Multiple session early psychological interventions for the prevention of post-traumatic stress disorder. Cochrane Database Syst Rev 2019;8:CD006869.

71 Luber M. EMDR with first responders: models, Scripted protocols, and summary sheets for mental health interventions. New York, NY: Springer Publishing, 2015

72 Jarero I, Artigas L. The EMDR protocol for recent critical incidents (EMDR-PRECl) and ongoing traumatic stress, 2015. Available: https://emdrfoundation.org/toolkit/preci.pdf

73 Shapiro E, Moench J. The EMDR group-traumatic episode protocol (G-TEP). presentation at the EMDR Canada annual conference, Québec City, Qc, 2018. Available: https://emdrresearchfoundation. org/toolkit/gtep.pdf

74 Schreiber M. Anticipate. Plan. Deter: BUILDING RESPONDER RESILIENCE INSTRUCTOR Guide [PowerPoint presentation], 2015. Available: http://file.lacounty.gov/SDSInter/dhs/221064_AnticipatePI anandDeterInstructorManual-FINAL.pdf

75 Mindarma. Mindarma.com, 2020. Available: https://www.mindarma. com/home/

76 Black Dog Institute. Mindarma workplace eLearning program - Black Dog Institute, 2020. Available: https://www.blackdoginstitute.org.au/ education-services/workplaces/workplace-programs/mindarma/

77 March on Stress. March on stress: TRIM FAQs, 2020. Available: http://www.marchonstress.com/page/p/trim_faqs

78 Operational Stress Management and Trauma Risk Management (TRiM) Cell - Royal Navy. The trauma risk management Handbook, 2020. Available: http://c69011.r11.cf3.rackcdn.com/d951c5627eb4 4b3789e84292d1e2c1fa-0x0.pdf

79 Hampshire Fire and Rescue Service and Hampshire Constabulary. The trauma risk management Handbook, 2020. Available: https:// documents.hants.gov.uk/corprhantsweb/Traumahandbook.pdf 